\title{
Probiotics and synbiotics targeting the intestinal microbiome attenuate non-alcoholic fatty liver disease
}

\author{
Luis Vitetta ${ }^{1,2}$, Jeremy D. Henson ${ }^{2,3}$ \\ ${ }^{1}$ Faculty of Medicine and Health, Sydney School of Medicine, The University of Sydney, Sydney, Australia; ${ }^{2}$ Medlab Clinical Sydney, Sydney, \\ Australia; ${ }^{3}$ Faculty of Medicine, Prince of Wales Clinical School, University of New South Wales, Sydney, Australia \\ Correspondence to: Prof. Luis Vitetta. 66 McCauley St., Alexandria, Sydney, New South Wales, Australia. Email: luis.vitetta@sydney.edu.au. \\ Provenance and Peer Review: This article was commissioned by the editorial office, Hepatobiliary Surgery and Nutrition. The article did not undergo \\ external peer review. \\ Comment on: Sharpton SR, Maraj B, Harding-Theobald E, et al. Gut microbiome-targeted therapies in nonalcoholic fatty liver disease: a systematic \\ review, meta-analysis, and meta-regression. Am J Clin Nutr 2019;110:139-49.
}

Submitted Oct 15, 2019. Accepted for publication Nov 12, 2019.

doi: 10.21037/hbsn.2019.11.24

View this article at: http://dx.doi.org/10.21037/hbsn.2019.11.24

Non-alcoholic fatty liver disease (NAFLD) presents a chronic condition that links the overarching influence of the intestinal microbiome to a disease state with the multisystem manifestations of obesity, type 2 diabetes (T2D), chronic kidney disease (CKD) and cardiovascular disease (CVD) (1). NAFLD is an increasingly common disease with a current prevalence of $25 \%$ worldwide (2). The proportion of severe presentations of NAFLD is also increasing and these trends will lead to an increased burden from NAFLD sequelae, including cirrhosis, liver failure and hepatocellular cancer (2).

The diverse community of microbes encountered in the intestines highlights the complexity confronting research of the gut-liver axis. Interrogating the intestinal microbiome while building an evidenced based approach for novel treatment modalities that target the gut cohort of bacteria focuses research expectations at reversing or inhibiting the progression of NAFLD pathogenesis. Preclinical studies employing gut microbiota transplants in germ-free animal models have demonstrated that the development of fatty liver disease is substantially determined by gut bacteria (3). Subsequently, clinical studies report that pro-inflammatory intestinal bacteria from the phylum Proteobacteria and the genus Escherichia coli predominate in patients diagnosed with NAFLD (4). In contrast, beneficial bacteria that can attenuate fatty liver pathology such as Faecalibacterium prausnitzii were reported decreased in NAFLD patients (4). Additional studies (5) that investigated the severity of liver disease in NAFLD correlated with shifts in intestinal microbiome patters such as increased Bacteroides, decreased Prevotella abundance and with increased abundance of Ruminococcus in NAFLD with fibrosis (5).

One mechanism postulated to link the gut microbiome with NAFLD is disruption of the gut epithelial barrier allowing microbial products to enter the portal circulation. Endotoxins from Gram-negative bacteria [e.g., lipopolysaccharides (LPS) from Escherichia coli] can induce intestinal inflammation and increase permeability, facilitating its own access to the liver where it can directly cause NAFLD and liver fibrosis (Figure 1) (1). In contrast, other bacterial products are needed to prevent inflammation via the nucleotide-binding oligomerisation domaincontaining protein 2 (a pattern recognition receptor involved in innate bacterial immunity) (6). Intestinal derived bacterial metabolites [such as butyrate and other short chain fatty acids (SCFA)] are also important signalling molecules for maintaining the epithelial cell-to-cell tight junction protein network and secretion of mucin proteins. Intestinal dysbiosis can lead to decreases in these metabolites and disruption of the intestinal epithelial barrier (7).

NAFLD can be considered a link between dysbiosis and a multisystem disease that includes CVD, CKD, T2D and obesity. Choline metabolism has been postulated as one mechanism behind this. Overgrowth of cholinemetabolising bacteria can cause choline deficiency, a known cause of NAFLD (8). A bacterial metabolite of 


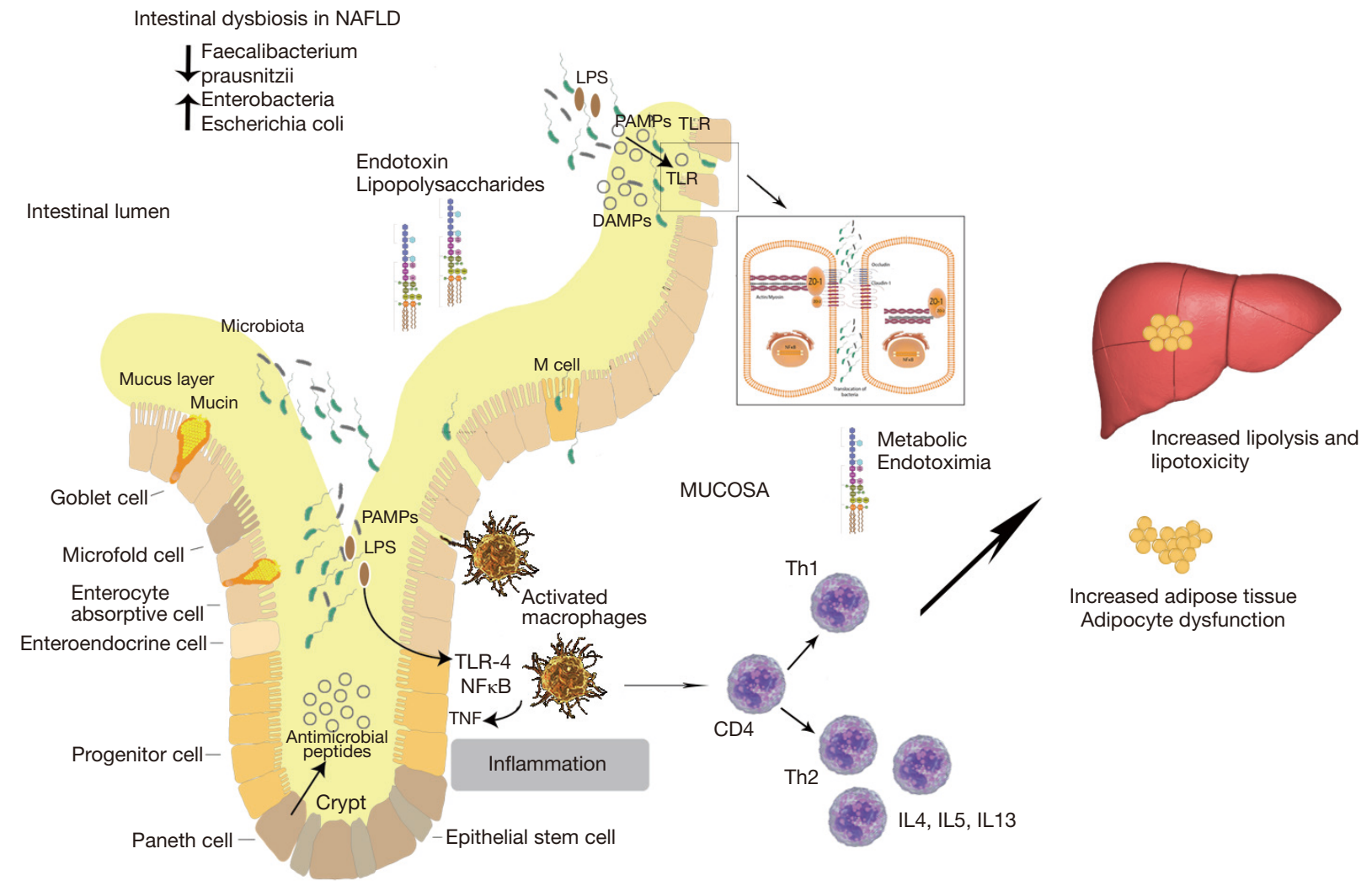

Figure 1 Intestinal mucosa inflammation links gut dysbiosis to a fatty liver [modified from Saltzman et al., 2018 (1)].

choline, trimethylamine is metabolised by the liver into trimethylamine-N-oxide, elevated levels of which is associated with CVD (8). LPS and SCFA also provide a link between the multisystem manifestations of NAFLD. LPS has been implicated as an inductor of a pro-inflammatory environment which is conducive to metabolic syndrome, insulin resistance and T2D. SCFA are important signalling molecules with systemic effects on metabolic regulation (7).

A compromised intestinal barrier can be associated with bacterial translocation from the gut into the systemic circulation increasing the risk of sepsis. LPS, a constituent of Gram-negative bacteria, is found to be increased in the systemic circulation, indicative of dysbiosis (9). LPS has been associated with inducing apoptosis of lymphocytes under in vivo conditions (10) demonstrating an immune-modulatory effect. Studies have suggested that a loss of lymphocytes in the intestinal mucosa is a consequence of intestinal epithelial dysbiosis and subsequent release of metabolic endotoxins (11). Gram-negative bacteria containing LPS are therefore hypothesized to contribute to NAFLD development. In addition dysbiosis and elevated systemic LPS can be predicted as markers of intestinal toxicity (12). Intestinal toxicity driven dysbiosis supports gut mucosal inflammatory responses that are concomitant with an increase in gut permeability. This combined disturbance of the intestinal barrier and local mucosal immune system activity can support and mediate NAFLD pathogenesis via the gut-liver axis (1).

The idea that a probiotic bacterium could improve inflammatory responses in the gut was demonstrated in a study that reported a decrease in hepatic steatosis with a probiotic that resulted in an analogous anti-inflammatory effect as anti-TNF antibody treatment (13). This result has provided a biologically plausible posit for the administration of probiotics to ameliorate NAFLD.

Numerous clinical trials have ensued, and Sharpton and colleagues' have provided a recent high quality systematic and meta-analysis study (14), published in the American Journal of Clinical Nutrition investigating the feasibility of probiotics and synbiotics administered as microbiome targeted therapies for the attenuation of NAFLD. The strength of the systematic review rests in the most current evidence of the included studies, which consisted of twenty- 
one randomized clinical trials of probiotics $(n=9)$ or synbiotics (i.e., a formulation that comprises probiotics + prebiotics; $n=12$ ) versus a comparator treatment. The most common comparator treatment was the use of a placebo in 14 trials, usual care in 5 and placebo + sitagliptin (both groups) in 1 and placebo + metformin (both groups) in 1 other study. There was high variability in the constituents of the formulations and clinical studies investigated.

Furthermore, studies were grouped according to the intervention and study outcome that allowed for clinical study heterogeneity assessment. Consequently, statistical heterogeneity was clearly evident and appropriately the $\mathrm{I}^{2}$ statistic was measured to indicate the percentage of variance attributed to the clinical studies that were included in the meta-analysis. Probiotics and synbiotics administration showed significant reduced liver stiffness measurements by elastography (weighed mean difference of $-0.70 \mathrm{kPa}, 95 \%$ CI: $-1.0,-0.4 \mathrm{kPa}$ ); and alanine aminotransferase activity (weighted mean difference of $-11.23 \mathrm{U} / \mathrm{L}, 95 \% \mathrm{CI}:-15.02$, $-7.44 \mathrm{U} / \mathrm{L})$ with high pooled study heterogeneity ( $\mathrm{I}^{2}: 93.4 \%$ and $90.6 \%$, respectively). Both probiotics and synbiotics had increased odds for improvement of hepatic steatosis (i.e., reduced) by graded ultrasound (odds ratio: 2.40, $95 \%$ CI: $1.5,3.84)$. Overall the meta-analysis and metaregression showed that the use of probiotics or synbiotics presented with improvements in liver specific markers of hepatic inflammation, liver stiffness measurements and liver steatosis in patients diagnosed with NAFLD. Unquestionably, the results of Sharpton and colleagues' meta-analysis study (14) should prompt and warrant more robust and well-defined trials.

Probiotics or synbiotics are not a panacea for the overall amelioration of chronic diseases but rather an evolving intestinal targeted therapy. The further development of probiotic/synbiotic based therapies for the prevention of progression and treatment of NAFLD requires a further focused molecular understanding of the multiple actions of probiotics and synbiotics in the intestines. Namely, such as the preservation of intestinal epithelia tight junctions as demonstrated in animal models of obesity induced NAFLD (15); the attenuation of intestinal inflammation in NAFLD (15); and the intestinal microbiome as the metaanalysis by Sharpton et al. (14) has reported.

\section{Acknowledgments}

We thanks Mr. Hayden Wells for his assistance with the design of the figure.
Funding: None.

\section{Footnote}

Conflicts of Interest: Both authors have completed the ICMJE uniform disclosure form (available at http://dx.doi. org/10.21037/hbsn.2019.11.24). LV reports in addition, LV has a patent 2017101478 issued. LV and JDH participate in the Medlab Clinical's research on probiotics.

Ethical Statement: The authors are accountable for all aspects of the work in ensuring that questions related to the accuracy or integrity of any part of the work are appropriately investigated and resolved.

Open Access Statement: This is an Open Access article distributed in accordance with the Creative Commons Attribution-NonCommercial-NoDerivs 4.0 International License (CC BY-NC-ND 4.0), which permits the noncommercial replication and distribution of the article with the strict proviso that no changes or edits are made and the original work is properly cited (including links to both the formal publication through the relevant DOI and the license). See: https://creativecommons.org/licenses/by-nc$\mathrm{nd} / 4.0 /$.

\section{References}

1. Saltzman ET, Palacios T, Thomsen M, et al. Intestinal Microbiome Shifts, Dysbiosis, Inflammation, and Nonalcoholic Fatty Liver Disease. Front Microbiol 2018;9:61.

2. Friedman SL, Neuschwander-Tetri BA, Rinella M, et al. Mechanisms of NAFLD development and therapeutic strategies. Nat Med 2018;24:908-22.

3. Le Roy T, Llopis M, Lepage P, et al. Intestinal microbiota determines development of non-alcoholic fatty liver disease in mice. Gut 2013;62:1787-94.

4. Zhu L, Baker SS, Gill C, et al. Characterization of gut microbiomes in nonalcoholic steatohepatitis (NASH) patients: a connection between endogenous alcohol and NASH. Hepatology 2013;57:601-9.

5. Boursier J, Mueller O, Barret M, et al. The severity of nonalcoholic fatty liver disease is associated with gut dysbiosis and shift in the metabolic function of the gut microbiota. Hepatology 2016;63:764-75.

6. Rodriguez-Nunez I, Caluag T, Kirby K, et al. Nod2 and Nod2-regulated microbiota protect BALB/c mice from diet-induced obesity and metabolic dysfunction. Sci Rep 
2017;7:548.

7. Feng Y, Huang Y, Wang Y, et al. Antibiotics induced intestinal tight junction barrier dysfunction is associated with microbiota dysbiosis, activated NLRP3 inflammasome and autophagy. PLoS One 2019;14:e0218384.

8. Sharpton SR, Ajmera V, Loomba R. Emerging Role of the Gut Microbiome in Nonalcoholic Fatty Liver Disease: From Composition to Function. Clin Gastroenterol Hepatol 2019;17:296-306.

9. Boulange CL, Neves AL, Chilloux J, et al. Impact of the gut microbiota on inflammation, obesity, and metabolic disease. Genome Med 2016;8:42.

10. Nielsen JS, Larsson A, Ledet T, et al. Rough-Form Lipopolysaccharide Increases Apoptosis in Human CD4(+) and CD8(+) T Lymphocytes. Scand J Immunol 2012;75:193-202.

11. Jiang $\mathrm{W}, \mathrm{Wu} \mathrm{N}, \mathrm{Wang} X$, et al. Dysbiosis gut microbiota

Cite this article as: Vitetta L, Henson JD. Probiotics and synbiotics targeting the intestinal microbiome attenuate non-alcoholic fatty liver disease. HepatoBiliary Surg Nutr 2020;9(4):526-529. doi: 10.21037/hbsn.2019.11.24 associated with inflammation and impaired mucosal immune function in intestine of humans with nonalcoholic fatty liver disease. Sci Rep 2015;5:8096.

12. Nolan JP. The role of intestinal endotoxin in liver injury: a long and evolving history. Hepatology 2010;52:1829-35.

13. Li Z, Yang S, Lin H, et al. Probiotics and antibodies to TNF inhibit inflammatory activity and improve nonalcoholic fatty liver disease. Hepatology 2003;37:343-50.

14. Sharpton SR, Maraj B, Harding-Theobald E, et al. Gut microbiome-targeted therapies in nonalcoholic fatty liver disease: a systematic review, meta-analysis, and metaregression. Am J Clin Nutr 2019;110:139-49.

15. Briskey D, Heritage M, Jaskowski LA, et al. Probiotics modify tight-junction proteins in an animal model of nonalcoholic fatty liver disease. Therap Adv Gastroenterol 2016;9:463-72. 